\title{
Construção de consenso Delphi das competências otorrinolaringológicas preconizadas ao egresso de Medicina
}

\author{
Building a Delphi consensus on Otorhinolaryngological skills for the medical graduate \\ Carolina de Castro Santos Rodrigues ${ }^{1}$ (D) | carolcastrorodrigues@hotmail.com \\ Ruth Borges Dias' (D) ruthdias@globo.com \\ Antônio Carlos Toledo Junior ${ }^{1}$ (D) $\mid$ toledoac@task.com.br
}

\begin{abstract}
RESUMO
Introdução: Afecções otorrinolaringológicas são destaques entre as enfermidades mais frequentes na atenção primária. Acredita-se que a sobrecarga na atenção secundária seja consequência da baixa resolução dos problemas na atenção primária. Uma possível explicação para esse fato seria a deficiência na capacitação médica durante a graduação. Estima-se que a carga horária média de otorrinolaringologia seja $0,6 \%$ da carga horaria média total, após análise de 141 matrizes curriculares, correspondendo a aproximadamente 70,5\% do total das escolas médicas em funcionamento em 2013. Nessa área, poucos estudos têm sido realizados em relação ao ensino e à necessidade de reavaliação curricular.
\end{abstract}

Objetivo: O presente estudo tem o intuito de buscar um consenso sobre as competências necessárias ao generalista na especialidade de otorrinolaringologia.

Métodos: Criou-se um questionário inicial que abordava as competências otorrinolaringológicas pertinentes à prática clínica dos médicos da atenção primária. Por meio da metodologia Delphi, no formato eletrônico, o questionário foi enviado para 20 especialistas com formações distintas: médicos generalistas, otorrinolaringologistas e médicos de família e comunidade. Essa heterogeneidade entre os especialistas contribuiu para garantir a confiabilidade dos resultados. Os resultados obtidos após cada rodada eram analisados pelos pesquisadores, que observavam as tendências e as opiniões dissonantes, bem como suas justificativas. Ao final da sistematização e compilação dos resultados, um novo questionário era elaborado e reenviado, iniciando uma nova rodada até que o consenso fosse estabelecido em todas competências.

Resultados: Realizaram-se cinco rodadas para o estabelecimento do consenso em todas as 17 competências otorrinolaringológicas avaliadas pelas proposições, o possibilitou a definição do nível de competência dos conteúdos e procedimentos otorrinolaringológicos preconizados ao egresso de Medicina.

Conclusão: Os dados obtidos neste trabalho podem servir para o embasamento, direcionamento e desenvolvimento do currículo otorrinolaringológico nos cursos de graduação de Medicina, visto que não se encontrou na literatura consenso estabelecendo as competências mínimas otorrinolaringológicas na formação curricular da graduação.

Palavras-chave: Consenso; Otorrinolaringologia; Atenção Primária; Educação Médica; Currículo.

\begin{abstract}
Introduction: Otorhinolaryngological disorders are amongst the most prominent frequent diseases in primary care. The overload in secondary care is thought to be a consequence of the low resolution of these problems in primary care. A deficiency in undergraduate medical training may explain this fact. The average estimated time spent studying otorhinolaryngological practice is estimated to be $0.6 \%$ of the total average practice hours after analysis of data from 141 medical course syllabuses in Brazil, corresponding to approximately $70.5 \%$ of all the medical schools in operation in 2013. Few studies have been conducted in this area and regarding teaching and the need for curriculum reassessment.

Objectives: This study seeks to ascertain a consensus on the skills required by the general practitioner in the specialty of otorhinolaryngology.

Methods: An initial questionnaire was devised addressing the otorhinolaryngological skills relevant to primary care clinical practice. Using the Delphi method, the questionnaire was sent in electronic format to 20 specialists with training in three different specialties; this heterogeneity of the survey sample helped ensure the reliability of the results. The results obtained after each round were analyzed by one researcher and validated by another, observing any discrepant trends and opinions, as well as their justifications. Once the results had all been compiled and systematized, a new questionnaire was devised and sent out, starting a new round until consensus had been established for all the skills.
\end{abstract}

Results: Five rounds were completed until a consensus was established for all 17 otorhinolaryngological skills evaluated by the propositions.

Conclusions: The data obtained by this work can serve as a basis and guideline for developing an otorhinolaryngological curriculum for undergraduate medical training since no consensus was found in the literature establishing such a minimum skill set.

Keywords: Medical Education; Curriculum; Consensus; Otorhinolaryngology; Primary Care.

1 Universidade José do Rosário Vellano, Belo Horizonte, Minas Gerais, Brasil.

Editora-chefe: Rosiane Viana Zuza Diniz.

Editora associada: Rosiane Viana Zuza Diniz.

Recebido em 04/02/21; Aceito em 28/04/21.

Avaliado pelo processo de double blind review. 


\section{INTRODUÇÃO}

O desafio da formação de médicos para as necessidades da população e dos sistemas de saúde vem sendo discutido no Brasil e em muitos outros países. Em particular, a adequação da formação médica voltada para a atenção primária à saúde tem sido alvo de inúmeros questionamentos em publicações, reuniões e congressos. A otorrinolaringologia é uma especialidade que envolve conhecimentos e habilidades clínicas em procedimentos e cirurgia, algumas das quais são essenciais na formação geral e para prover atenção à saúde a adultos e crianças ${ }^{1}$.

\section{Importância das afecções otorrinolaringológicas na atenção primária}

As afecções otorrinolaringológicas destacam-se entre as enfermidades mais presentes na atenção primária. Quando se avalia a classificação internacional de atenção primária, observa-se que, entre os 28 problemas de saúde mais frequentes, estão descritas quatro afecções relacionadas à otorrinolaringologia, sendo elas: infecções agudas do aparelho respiratório superior, amigdalite aguda, otite média aguda e rinite alérgica, correspondendo a $6,1 \%$ de todos os problemas correlacionados. Quando se observam especificamente os 30 motivos das consultas mais frequentes, identificam-se vários sintomas correlacionados às afecções otorrinolaringológicas, como tosse, febre, dor de garganta, dificuldade respiratória, vertigens, tonturas e dor de ouvido, o que corresponde a $10,4 \%$ de todas as estratificadas na classificação internacional da atenção primária².

Outros trabalhos estimam que afecções do ouvido, do nariz e da garganta correspondam a um índice que varia de $20 \%$ a $25 \%$ dos casos atendidos na atenção primária à saúde, chegando a até $50 \%$ dos atendimentos pediátricos ${ }^{1}$.

\section{Otorrinolaringologia e ensino nas faculdades de Medicina}

Uma possível explicação para a baixa resolutividade da atenção primária em relação às afecções otorrinolaringológicas é a deficiência da capacitação dos médicos generalistas na graduação. Em geral, o ensino em otorrinolaringologia restringe-se aos anos finais do curso de graduação, sendo em sua essência teórico. Estima-se que a carga horária média de otorrinolaringologia seja $0,6 \%$ da carga horaria média total, após análise de 141 matrizes curriculares de cursos de Medicina do Brasil, correspondendo a aproximadamente $70,5 \%$ do total das escolas médicas em funcionamento em 2013'.

Considerando que a incidência de afecções otorrinolaringológicas é alta na população, torna-se desejável que parte da carga horária reservada à otorrinolaringologia seja aproveitada em atividades de ensino prático, como a realização adequada de exame otorrinolaringológico básico e a capacitação em realizar diagnósticos diferenciais de doenças relativamente simples e comuns, como um quadro gripal e uma sinusite aguda, ou quando caracterizar uma situação de urgência ${ }^{3}$.

\section{Treinamento educacional baseado em competências}

A inconsistência na definição e estruturação das competências e a falta de uma abordagem e linguagem padronizadas em relação a elas dificultam sua adoção universal na educação ${ }^{4}$. A educação baseada em competências visa a níveis padronizados de proficiência, com o objetivo de garantir que todos os alunos alcancem determinado nível de proficiência na conclusão do treinamento ${ }^{5}$. Portanto, as competências podem ser consideradas habilidades ou capacidades e são as unidades organizadoras do currículo médico baseado em competências ${ }^{6}$.

O trabalho holandês que estabeleceu o nível de competência dos alunos egressos do curso de Medicina recomendou que os médicos recém-formados sejam capazes de demonstrar as competências básicas na prática, o que significa ter conhecimentos, habilidades e comportamentos profissionais, e saber lidar adequadamente com problemas que envolvam altos níveis de complexidade. Nesse estudo, as competências foram estabelecidas em níveis que deverão ser alcançados pelos graduandos de Medicina, como demonstrado no Quadro 1, determinando que estes devam possuir, no mínimo, o nível II de competência no domínio das questões relacionadas ao processo de saúde e às doenças preconizados. No entanto, dependendo das especificidades e dos destaques dos programas de treinamento de cada faculdade, serão alcançados níveis de competência mais altos ${ }^{7}$.

Swensson', ao revisar a literatura sobre competências mínimas que envolvem aspectos da otorrinolaringologia, não encontrou consensos estabelecidos.

Nesse cenário da formação profissional no Brasil, considerando o campo de atuação, a formação do médico generalista e a relevância das afecções otorrinolaringológicas, este estudo objetiva construir um consenso sobre as competências em otorrinolaringologia necessárias ao médico generalista egresso da faculdade para atuação na atenção primária. Além disso, identificam-se as condições e as doenças relacionadas à otorrinolaringologia que devem ser ensinadas e define-se o nível de competência que os médicos generalistas e egressos do curso de Medicina devem ter em relação aos conhecimentos otorrinolaringológicos. 
Quadro 1. Definição dos níveis de competência.

\begin{tabular}{cl}
\hline Nível de competência & \multicolumn{1}{c}{ Descrição } \\
\hline 1 & $\begin{array}{l}\text { Possui conhecimento e compreensão dos ramos da ciência relevantes à medicina. } \\
\text { Demonstra em situações padronizadas que possui habilidades relevantes à medicina. } \\
\text { Possui habilidades básicas necessárias ao comportamento profissional. }\end{array}$ \\
& $\begin{array}{l}\text { Possui conhecimentos, habilidades e comportamentos profissionais integrados, com abordagem } \\
\text { adequada às questões de doença e saúde incorporadas nessa estrutura, demonstrando essa } \\
\text { competência em treinamentos de situações contextualizadas. }\end{array}$ \\
& $\begin{array}{l}\text { Realiza adequadamente atividades profissionais definidas nas competências dos médicos em } \\
\text { treinamentos específicos ou situações profissionais simuladas. } \\
\text { Realiza adequadamente e de forma independente as atividades profissionais definidas nas } \\
\text { competências dos médicos em situaços profissionais autênticas. } \\
\text { Recebe instruções prévias específicas de um praticante experiente. } \\
\text { Realiza adequadamente e de forma independente as atividades profissionais definidas nas } \\
\text { competências dos médicos em situaçoses profissionais autênticas. } \\
\text { Um praticante experiente é disponibilizado em stand-by, fornecendo supervisão após o evento. }\end{array}$ \\
&
\end{tabular}

Fonte: Laan et al.7.

\section{MATERIAIS E MÉTODOS}

\section{Desenho do estudo}

O estudo utilizou da metodologia Delphi que permite reunir um conjunto de opiniões de especialistas separados geograficamente, levando a resultados densos sobre temáticas complexas e abrangentes ${ }^{8}$. Foi utilizada a metodologia Delphi no formato eletrônico, mantendo-se os preceitos básicos da metodologia original: o anonimato dos respondentes, a representação estatística da distribuição dos resultados e o feedback de respostas do grupo para reavaliação das rodadas subsequentes que ocorreram até o consenso ser alcançado ${ }^{9-11}$. O cálculo do número de especialistas encontrado na literatura é muito variado, mas os estudos indicam que um número ótimo deles não deve ser inferior a dez, pois compromete os resultados em termos de consenso efetivo e relevância das informações obtidas. No entanto, um número muito elevado gera uma quantidade enorme de dados e torna a administração e a análise muito complexas, além de não proporcionar a produção de novas ideias ${ }^{12}$. Em um grupo Delphi, o mais importante é o equilíbrio das participações, em vez do tamanho, representado pela miríade de pontos de vista, expertises e interesses no contexto $^{13}$. Estudos apontam que entre dez e 18 especialistas é o quantitativo mais adequado para desenvolver o método ${ }^{14}$.

\section{Participantes}

A amostra selecionada foi do tipo não probabilística, realizada por conveniência, definida por acessibilidade, notório saber e interesse pelo tema dentro do território nacional, por meio de contatos interpessoais. Participaram da pesquisa 20 médicos, $10 \%$ a mais do máximo sugerido ${ }^{14}$, visando minimizar impactos negativos de possíveis perdas ao longo das rodadas de perguntas e respostas previstas no processo do método Delphi.
A amostra foi composta por sete especialistas em medicina de família e comunidade, seis médicos generalistas com experiência em clínica médica e/ou experiência comprovada na atenção primária e sete otorrinolaringologistas.

Utilizaram-se os seguintes critérios de inclusão: otorrinolaringologistas - membros da Sociedade Brasileira de Otorrinolaringologia -, todos devidamente titulados e registrados como especialistas no Conselho Regional de Medicina, obedecendo ao critério mínimo de cinco anos de experiência e atuação na área, podendo ser docente ou não; especialistas em medicina de família e comunidade - membros da Sociedade Brasileira de Medicina de Família e Comunidade -, todos devidamente titulados e registrados como especialistas no Conselho Regional de Medicina, obedecendo ao critério mínimo de cinco anos de experiência e atuação na área, podendo ser docente ou não; e médicos generalistas - atuantes no Sistema Único de Saúde -, formados há, no mínimo, quatro anos, com experiência em clínica médica e/ou experiência comprovada na atenção primária.

\section{Coleta e análise de dados}

O trabalho foi iniciado com o envio de questionário on-line, criado na plataforma Google Forms. O instrumento abordava, por meio de afirmativas, as competências otorrinolaringológicas pertinentes à prática clínica dos médicos da atenção primária, tendo em vista o currículo baseado em competências para medicina de família e comunidade. O referido currículo é fruto do trabalho de um ano com a participação de dezenas de especialistas com experiência no tema no Brasil e no exterior ${ }^{15}$.

O processo de construção do currículo foi dividido em quatro etapas. A primeira etapa contou com a presença de um 
grupo de consultores canadenses vinculados à Universidade de Toronto e um grupo de especialistas brasileiros com atuação e formação em medicina de família e comunidade. O produto dessa oficina, que durou cinco dias, foi compilado, organizado e padronizado em formato de planilhas divididas por campos e áreas de competência. A segunda etapa consistiu na revisão do conteúdo, utilizando a metodologia Delphi, e contou com a participação de especialistas com experiência e formação médica. Ao final das rodadas de revisão sequencial, o material foi novamente compilado e organizado. A terceira etapa possibilitou uma consulta pública, de modo que o material resultante da oficina e das revisões foi disponibilizado ao público e colocado em consulta durante todo o mês de dezembro de 2014. A quarta etapa facilitou o painel com os especialistas, contando com um grupo de especialistas na área, com experiência em formação médica em medicina de família e comunidade. Essa última etapa foi destinada a avaliar as contribuições decorrentes da consulta pública, fazendo a revisão final do material e padronizando a linguagem utilizada ${ }^{15}$.

A seleção de bibliografia basilar para o presente estudo respaldou-se na tendência atual de políticas públicas que incentivam o desenvolvimento desse nível de assistência e do movimento de inserção precoce do aluno na atenção primária'. Por se tratar de documento direcionado para residência de medicina de família e comunidade, na elaboração do questionário, foram consideradas as competências otorrinolaringológicas classificadas no currículo baseado em competências para medicina de família e comunidade como pré-requisitos essenciais e desejáveis, descartando as competências consideradas avançadas. Após ser validado por teste-piloto, respondido na presença do pesquisador por dez médicos generalistas que não faziam parte do grupo dos especialistas selecionados para participação do trabalho, avaliaram-se a aplicabilidade do questionário, o tempo médio de preenchimento, a dificuldade de compreensão, a clareza e a objetividade dos itens.

O primeiro questionário, composto por 16 itens, foi respondido individualmente pelos participantes, e os resultados obtidos na primeira rodada foram analisados quantitativa e qualitativamente pelo pesquisador e revisado por outro pesquisador, observando as tendências e as opiniões dissonantes, bem como suas justificativas, sistematizando-as e compilando-as para, posteriormente, reenviá-las ao grupo por meio de novo questionário composto por 17 proposições, porque um item foi desmembrado em dois, conforme solicitação dos participantes. Segundo Black, quando se atinge convergência nas respostas igual ou superior a $75 \%$, considera-se que houve consenso para inclusão ou exclusão de um tópico ${ }^{11}$. Embasando-se na escala de valoração tipo
Likert $^{16}$, que vai de 1 (discordância máxima) a 6 (concordância máxima), utilizaram-se, neste trabalho, o critério de exclusão da proposição, quando a convergência das respostas nos números 1 e 2 foi igual ou superior a 75\%, e o critério de inclusão da proposição, quando a convergência das respostas nos números 5 e 6 foi igual ou superior a 75\% ${ }^{11,16}$. As proposições que não atingiram esse critério foram reelaboradas pelo primeiro pesquisador e validadas por outro pesquisador, levando em consideração os comentários e as respostas dos especialistas. O processo se repetiu, em rodadas sucessivas, até que o consenso foi alcançado em todas as 17 proposições.

A implementação do método Delphi se deu em 18 etapas que foram divididas em:

- construção do primeiro questionário abordando conhecimentos otorrinolaringológicos pertinentes à prática clínica dos médicos da atenção primária, fundamentado no currículo baseado em competências para medicina de família e comunidade $^{15}$;

- execução do teste-piloto do primeiro questionário;

- seleção do grupo de especialistas em medicina de família e comunidade, otorrinolaringologistas e médicos generalistas aptos segundo critérios de inclusão;

- contato com os especialistas selecionados, convite para participação na pesquisa e envio do primeiro questionário;

- recebimento das respostas do primeiro questionário;

- análise qualitativa e quantitativa das respostas do primeiro questionário;

- construção e envio do segundo questionário com feedback baseado na análise das respostas do primeiro questionário;

- recebimento das respostas do segundo questionário;

- análise qualitativa e quantitativa das respostas do segundo questionário;

- construção e envio do terceiro questionário com feedback baseado na análise das respostas do segundo questionário;

- recebimento das respostas do terceiro questionário;

- $\quad$ análise qualitativa e quantitativa das respostas do terceiro questionário;

- construção e envio do quarto questionário com feedback baseado na análise das respostas do terceiro questionário;

- recebimento das respostas do quarto questionário;

- $\quad$ análise qualitativa e quantitativa das respostas do quarto questionário; 
- construção e envio do quinto questionário com feedback baseado na análise das respostas do quarto questionário;

- recebimento das respostas do quinto questionário;

- análise qualitativa e quantitativa das respostas do quinto questionário;

- $\quad$ final do processo, escrita das conclusões e consensos.

A análise qualitativa e quantitativa dos resultados desta pesquisa forneceu as informações necessárias aos pesquisadores para o desenvolvimento e estabelecimento dos níveis de competência preconizados aos egressos do curso de Medicina, com relação aos conteúdos otorrinolaringológicos contemplados no consenso. Esse processo de elaboração foi realizado em duas etapas. Na primeira etapa, o primeiro pesquisador elaborou individualmente um esboço dos níveis de competências preconizados aos egressos do curso de Medicina, fundamentando-se nos dados extraídos nas sucessivas rodadas realizadas na metodologia Delphi, necessárias para o estabelecimento do consenso deste trabalho, respaldando-se no modelo sugerido por Laan et al. ${ }^{7}$, descrito no Quadro 1. A segunda etapa consistiu na contraposição e validação do material elaborado na primeira etapa pelo segundo pesquisador.

Para o desenvolvimento da pesquisa, os princípios de bioética foram respeitados, em concordância com a Resolução no 510/2016 da Comissão Nacional de Ética em Pesquisa do Ministério da Saúde, que dispensa o uso de Termo de Consentimento Livre e Esclarecido (TCLE) por se tratar de pesquisa de opinião.

\section{RESULTADOS}

O presente estudo foi realizado de 2019 a 2020 e contou com a participação de 20 médicos de ambos os sexos, sendo $55 \%$ do sexo feminino e $45 \%$ do sexo masculino, com idade entre 31 e 62 anos, idade média de 40 anos. O tempo de experiência profissional variou de quatro a 38 anos, com média de 16 anos. A média do exercício da especialidade entre otorrinolaringologistas e médicos da família e comunidade foi de 12 anos, variando de 5 a 34 anos. A amostra dos participantes foi composta por médicos que residem e trabalham no interior de Minas Gerais e São Paulo, assim como médicos que residem e trabalham nas capitais dos estados de Minas Gerais, São Paulo, Espírito Santo e Paraná.

$\mathrm{Na}$ primeira rodada, os 20 especialistas selecionados que aceitaram fazer parte do trabalho tiveram como instrução avaliar as competências otorrinolaringológicas abordadas nas 16 proposições do questionário. Na primeira rodada, todos os 20 participantes responderam ao questionário, e as proposições que não atingiram o consenso foram reformuladas, observando as respostas e os comentários apontados pelos participantes. Uma proposição foi desmembrada em duas proposições distintas objetivando avaliar separadamente as competências de otite média crônica e otite média serosa. Não houve sugestões de inclusão de outros itens abordando competências diferentes das propostas pelo questionário. Dezoito especialistas responderam ao segundo questionário, e, entre os dois especialistas que não responderam a esse questionário, um era otorrinolaringologista, e o outro, médico de família e comunidade. Na terceira rodada, 19 especialistas responderam ao questionário, e o participante que não o fez era médico de família e comunidade. Dezoito dos especialistas selecionados responderam à quarta rodada, e, entre os participantes que não responderam, um era otorrinolaringologista, e o outro, médico de família e comunidade. Dezoito especialistas selecionados responderam à quinta rodada, e os participantes que não o fizeram eram médicos de família e comunidade. Alcançou-se o consenso no quinto questionário, e as rodadas de perguntas e respostas foram encerradas após a sua análise. As instruções, os critérios de análise e a metodologia da pesquisa não sofreram modificações durante as rodadas.

Tabela 1. Consenso das competências otorrinolaringológicas.

Competência otorrinolaringológica
Nível de concordância para inclusão

75,0\% Primeira

$88,0 \%$

Segunda

2) Avaliar corpo estranho de ouvido e remover os que são factíveis com a lavagem de conduto auditivo externo. Encaminhar para assistência especializada os casos com lacerações importantes de conduto auditivo externo e/ou o corpo estranho de difícil remoção.

3) Conhecer os principais tipos de labirintopatias e saber tratar as mais simples. Encaminhar para a atenção secundária as mais complexas ou refratárias ao tratamento proposto na atenção primária.
$83,3 \%$

Segunda 
Tabela 1. (Continuação) Consenso das competências otorrinolaringológicas.

Competência otorrinolaringológica
Nível de concordância para inclusão
Rodada do consenso

4) Orientar e avaliar clinicamente os pacientes com tinnitus, descartando distúrbios endócrino-metabólicos e tratando as comorbidades na atenção primária. Sendo responsabilidades do especialista a avaliação auditiva, o $83,3 \%$ Quarta estabelecimento do diagnóstico e a programação terapêutica.

5) Realizar ação preventiva relacionada ao câncer de orofaringe na atenção primária.

$\begin{array}{lr}85,0 \% & \text { Primeira } \\ 94,4 \% & \text { Segunda } \\ 90,0 \% & \text { Primeira } \\ 90,0 \% & \text { Primeira } \\ 77,7 \% & \text { Quarta }\end{array}$

6) Realizar a abordagem inicial do controle da epistaxe. Encaminhar o paciente para o serviço especializado nos casos de epistaxe volumosa ou refratária.

7) Realizar otoscopia e saber identificar perfuração da membrana timpânica.

8) Saber diferenciar e saber tratar otite média aguda e otite externa.

9) Solicitar audiometria na atenção primária. Encaminhar para o especialista os casos alterados para investigação, estabelecimento do diagnóstico e programação terapêutica.

10) Ter conhecimento sobre a clínica, os sinais e os sintomas da presbiacusia, suspeitar do diagnóstico e solicitar a avaliação auditiva. Encaminhar para o especialista para estabelecimento do diagnóstico e dos critérios para adaptação de Aasi*.

11) Identificar hipertrofia de adenoide e/ou amígdala.

12) Diagnosticar e tratar rinossinusites agudas.

13) Saber diagnosticar as rinites e saber tratar os casos de rinite intermitente e persistente leve. Encaminhar para atenção secundária os casos de rinite persistente moderada e grave.

14) Fazer diagnóstico de rinossinusite crônica. Encaminhar para atenção secundária para confirmação diagnóstica e tratamento.

Quinta

15) Ter conhecimento sobre a clínica, os sinais e os sintomas da otite média crônica. Encaminhar os casos suspeitos para a atenção secundária para confirmação de diagnóstico e tratamento.

16) Ter conhecimento sobre a clínica, os sinais e os sintomas da otite média serosa em crianças e adultos. Encaminhar ao especialista os casos suspeitos para confirmação do diagnóstico e tratamento.

75,0\% Primeira

90,0\% Primeira

$94,4 \% \quad$ Segunda

77,7\% Segunda

78,9\% Terceira

17) Ter conhecimento sobre a clínica, os sinais e os sintomas do desvio de septo nasal obstrutivo. Encaminhar para avaliação especializada os pacientes com anamnese e exame físico compatíveis para diagnóstico e conduta.

*Aasi: aparelho de amplificação sonora individual.

Quadro 2. Definição dos níveis de competência em otorrinolaringologia para egressos de cursos de Medicina.

\begin{tabular}{lc}
\hline \multicolumn{1}{c}{ Competência otorrinolaringológica } & Nível de competência \\
\hline Realizar controle inicial de epistaxe. & 2 \\
Suspeitar das principais hipóteses diagnósticas das labirintopatias. & 2 \\
Suspeitar das principais hipóteses diagnósticas de tinnitus. & 2 \\
Suspeitar dos principais diagnósticos de perdas auditivas. & 2 \\
Suspeitar do diagnóstico da presbiacusia. & 2 \\
Suspeitar do diagnóstico da rinossinusite crônica. & 2 \\
Suspeitar do diagnóstico da otite média crônica. & 2 \\
Suspeitar do diagnóstico de otite média serosa em crianças e adultos. & 2 \\
Suspeitar do diagnóstico de obstrução nasal secundária ao desvio septal. & 2 \\
Realizar remoção de cerume. & 3 \\
Realizar remoção de corpo estranho de ouvido. & 3 \\
\hline
\end{tabular}


Quadro 2. (Continuação) Definição dos níveis de competência em otorrinolaringologia para egressos de cursos de Medicina.

\begin{tabular}{lc}
\multicolumn{1}{c}{ Competência otorrinolaringológica } & Nível de competência \\
\hline Realizar avaliação preventiva relacionada ao câncer de orofaringe. & 3 \\
Estabelecer diagnóstico de obstrução de via aérea superior secundária à hipertrofia de & 3 \\
adenoide e/ou amígdala. & 3 \\
Tratar comorbidades e disfunções endocrinometabólicas dos pacientes com tontura e tinnitus. & 4 \\
Realizar otoscopia. & 4 \\
Estabelecer diagnóstico e tratar obstrução nasal secundária ao processo inflamatório causado & 4 \\
pela rinite intermitente e persistente leve. & 4 \\
Estabelecer diagnóstico e tratar otite média aguda. & 4 \\
Estabelecer diagnóstico e tratar otite externa. & 4 \\
Estabelecer diagnóstico e tratar rinossinusite aguda. & 4 \\
\hline
\end{tabular}

\section{DISCUSSÃO}

Na formação de um grupo Delphi, o fator de maior relevância é o equilíbrio das participações, e não o tamanho ${ }^{13}$. Foram selecionados 20 especialistas, mantendo o equilíbrio quantitativo dos participantes de cada grupo, e não houve privilégio de nenhum grupo, ocorrendo equidade entre as manifestações de opiniões e participações dos representantes de cada grupo.

A comissão permanente de avaliação da Faculdade de Medicina da Universidade Federal de Minas Gerais, em busca do aperfeiçoamento do processo de ensino-aprendizagem do curso médico, realizou trabalho que objetivava o estabelecimento de matriz de competências essenciais para a formação do médico generalista ${ }^{17}$. No entanto, o trabalho citado não descreve e não estabelece os problemas mais prevalentes em otorrinolaringologia que deveriam ser destacados nesse domínio específico de conhecimento. Isso posto, o estabelecimento do consenso visa preencher essa lacuna, possibilitando aos educadores médicos o desenvolvimento de um trabalho mais direcionado e assertivo nessas áreas do conhecimento médico.

O trabalho de Swensson' ressalta que a obstrução nasal, a respiração bucal e os roncos são manifestações geralmente relacionadas à mesma patologia de base, sendo as situações otorrinolaringológicas mais encaminhadas ao especialista. Segundo Abreu et al. ${ }^{18}$, o diagnóstico da síndrome da respiração oral em crianças é essencialmente clínico, e muitas vezes o pediatra ou o médico da família e comunidade são os primeiros a ter contato com o paciente respirador oral, cabendo a esses profissionais a execução de anamnese dirigida e detalhada para o diagnóstico precoce e tratamento adequado. $O$ estudo de Guerra ${ }^{19}$ avaliou a capacidade resolutiva e a validade diagnóstica em doenças otorrinolaringológicas na atenção primária e comparou o diagnóstico das doenças otorrinolaringológicas mais comuns na atenção primária em relação ao diagnóstico referência estabelecido na atenção secundária para o mesmo paciente em questão. A concordância diagnóstica entre a atenção primária e a secundária foi de 31,6\%. O estudo concluiu que a maioria dos casos que foram encaminhados para o especialista poderiam ter sido resolvidos no nível primário de atenção à saúde. Como suscitado nos trabalhos descritos e evidenciado pelo resultado qualitativo desta pesquisa e pelo consenso estabelecido, o treinamento teórico e prático das afecções mais comuns na população é demasiadamente importante para o condicionamento do aluno, objetivando dessa forma gerar a capacidade de presumir e até mesmo estabelecer corretamente os diagnósticos das afecções otorrinolaringológicas mais comuns na atenção primária.

Swensson' sugere que situações que não fazem parte do nível primário de atendimento, como casos de epistaxes importantes, queixas que demandem realização de exames específicos e doenças que tenham cirurgias como forma de tratamento, serão vivenciadas pelos alunos nos outros níveis de atenção à saúde durante os anos de graduação, devendo-se dar enfoque à inserção precoce do aluno na atenção primária a saúde, quando terá a oportunidade de praticar o atendimento de vários problemas relacionados à otorrinolaringologia. Em concordância com o trabalho mencionado, o consenso estabelecido nesta pesquisa propõe que o conhecimento global nos conteúdos otorrinolaringológicos vistos como de competência do especialista também é importante para que o médico generalista suspeite do diagnóstico e referencie adequadamente o paciente, evitando que afecções importantes sejam subdiagnosticadas.

$\mathrm{Na}$ análise das argumentações dos participantes, observou-se que eles, ao refutarem a competência abordada na proposição, frequentemente usavam como justificativa a falta de treinamento e capacitação médica nas faculdades, o que pode ter limitado a discussão por um viés de tempo. $O$ trabalho buscava estabelecer um consenso das competências 
otorrinolaringológicas pertinentes ao egresso para sua prática profissional, com o objetivo de instituir e estratificar as habilidades que devem ser trabalhadas e desenvolvidas na graduação, pela relevância, não devendo restringir o debate à avaliação da realidade atual. Dessa forma, a percepção dos egressos atuais pode ter influenciado no processo de consenso. Outra limitação deste estudo está relacionada à ausência do estabelecimento de carga horária mínima curricular, assim como sua distribuição em aulas práticas e teóricas necessárias para que o aluno atinja as competências otorrinolaringológicas estabelecidas nesse consenso.

Por se tratar de tema relevante e pouco estudado no Brasil, são necessários novos estudos para a definição dos conteúdos essenciais de um currículo de otorrinolaringologia na graduação médica brasileira, assim como o estabelecimento de carga horária mínima de aulas teóricas e práticas, o que é preconizado para os egressos atingirem os níveis de competências propostos neste trabalho.

\section{CONCLUSÃO}

Por meio do consenso, observa-se a importância de ressaltar, direcionar e treinar adequadamente os alunos para os temas e procedimentos frequentes na prática clínica do generalista. Tudo isso visa evitar que ocorra, no âmbito da otorrinolaringologia, uma grande disparidade entre a carga curricular na escola médica e a importância desses problemas para o aluno da graduação, que deverá sair do curso capacitado para atuar como médico generalista. Deve-se seguir o consenso para impedir que ocorra situação inversa entre o tempo utilizado estudando uma doença em particular e a baixa incidência na população em geral. Deve-se ainda incentivar a dedicação de tempo para o aprendizado teórico e prático, objetivando atingir níveis mais altos de competências nas afecções mais comuns. Entre essas encontram-se o diagnóstico e o tratamento das rinossinusites agudas, otites externas, otites médias agudas, conhecimento das principais causas de obstrução nasal, assim como a realização da remoção de cerume e de corpo estranho de ouvido, além de ações preventivas de câncer de orofaringe.

\section{O conhecimento global em conteúdos} otorrinolaringológicos menos frequentes ou designados no consenso de trabalho como de competência do especialista também é necessário, no entanto exige níveis de competência mais baixos, sendo o nível 2 de competência (no qual o médico exibe conhecimentos, habilidades e comportamentos profissionais integrados, de modo a demonstrar habilidade e abordagem adequada em treinamentos de situações contextualizadas) suficiente para que o médico egresso suspeite do diagnóstico e referencie adequadamente o paciente, evitando que afecções importantes sejam subdiagnosticadas.

\section{CONTRIBUIÇÃO DOS AUTORES}

Todos os autores contribuíram igualmente.

\section{CONFLITO DE INTERESSES}

Declaramos não haver conflito de interesses.

\section{FINANCIAMENTO}

Declaramos não haver financiamento.

\section{REFERÊNCIAS}

1. Swensson RPA. Otorrinolaringologia na formação do médico e na atenção primária em saúde [dissertação]. Campinas: Universidade Estadual de Campinas; 2013.

2. Gusso GDF. Diagnóstico de demanda em Florianópolis utilizando a Classificação Internacional de Atenção Primária: 2 edição (CIAP-2) [tese]. São Paulo: Universidade de São Paulo; 2009.

3. Person OC, Mendonça RR, Yoshimura R, Abdo TRT, Mattiuz EBR, Rapoport PB. O ensino em otorrinolaringologia na graduação médica. Arq Méd ABC. 2004;29(2):91-4.

4. Hawkins RE, Welcher CM, Holmboe ES, Kirk LM, Norcini JJ, Simons KB, et al. Implementation of competency-based medical education: are we addressing the concerns and challenges? Med Educ. 2015;49:1086-102. doi: $10.1111 /$ medu.12831.

5. Ten Cate O. Entrustability of professional activities and competencybased training. Med Educ. 2005; 39(12):1176-7. doi: 10.1111/j.13652929.2005.02341.x.

6. Albanese MA, Mejicano G, Mullan P, Kokotailo P, Gruppen L. Defining characteristics of educational competencies. Med Educ. 2008;42(3):24855. doi: 10.1111/j.1365-2923.2007.02996.x.

7. Laan RF, Leunissen RR, van Herwaarden CL. The 2009 framework for undergraduate medical education in the Netherlands. GMS Z Med Ausbild. 2010;27(2):Doc35. doi: 10.3205/zma000672.

8. Linstone HA, Turoff M. The Delphi method: techniques and applications. New Jersey: Institute of Technology; 2002 [acesso em 21 jan 2021]. Disponível em: https://web.njit.edu/ turoff/pubs/delphibook/index.html.

9. Giovinazzo RA. Modelo de aplicação de metodologia Delphi pela internet: vantagens e ressalvas. Administração On Line, Rio de Janeiro. 2001;2(2) [acesso em 8 ago 2019]. Disponível em: http://www.fecap.br/adm_online/ art22/renata.htm.

10. Loures CAS. Delphi na internet e suas Implicações do ponto de vista metodológico. [S. I.], 2002 [acesso em 27 jan 2021]. Disponível em: http:// www.anpad.org.br/admin/pdf/enanpad2002-epa-1172.pdf.

11. Pope $C$, Mays N. Pesquisa qualitativa na atenção à saúde. 3a ed. Porto Alegre: Artmed; 2009.

12. Marques JBV, Freitas D. Método DELPHI: caracterização e potencialidades na pesquisa em educação. Pro-Posições. 2018;29(2):389-415. doi: 10.1590/1980-6248-2015-0140.

13. Bloor M, Sampson H, Baker S, Dahlgren K. Useful but no Oracle: reflections on the use of a Delphi Group in a multi-methods policy research study. Qual Res. 2015;15:57-70. doi: 10.1177/1468794113504103.

14. Boulkedid R, Abdoul H, Loustau M, Sibony O, Alberti C. Using and reporting the Delphi method for selecting healthcare quality indicators: a systematic review. PLoS One. 2011;6(6):e20476. doi: 10.1371/journal. pone.0020476.

15. Sociedade Brasileira de Medicina de Família e Comunidade. Currículo baseado em competências para medicina de família e comunidade. Rio de Janeiro: SBMFC; 2015 [acesso em 21 jan 2021]. Disponível em: https:// www.sbmfc.org.br/noticias/sbmfc-divulga-curriculo-baseado-emcompetencias/.

16. Grisham T. The Delphi technique: a method for testing complex and multifaceted topics. International Journal of Managing Projects in Business. 2009;2(1):112-30. doi: 10.1108/17538370910930545. 
17. Gontijo ED, Alvim C, Megale L, Melo FRC, Lima MECC. Matriz de competências essenciais para a formação e avaliação de desempenho de estudantes de medicina. Rev Bras Educ Med. 2013;37(4):526-39. doi: 10.1590/S0100-55022013000400008.

18. Abreu RR, Rocha RL, Lamounier JA, Guerra AFM. Etiologia, manifestações clínicas e alterações presentes nas crianças respiradoras orais. J Pediatr. 2008;84(6):529-35. doi: 10.1590/S0021-75572008000700010.
19. Guerra AFM. Capacidade resolutiva em otorrinolaringologia do médico da atenção primária da rede pública de saúde do município de Belo Horizonte [dissertação]. Belo Horizonte: Universidade Federal de Minas Gerais; 2006. 